\title{
Proving Gödel's Completeness and Incompleteness Theorems (in restricted cases) using $S d$ - Topology over the theory of a Model
}

\author{
Soham Dasgupta \\ (Ex. PGT (Math) at Kendriya Vidyalaya Ordnance Factory, Dumdum, Kolkata, W.B , INDIA) \\ Type: Research article ( Sub: Pure Mathematics)
}

AMS -MSC2010 No. 03B99

\begin{abstract}
This is a modification as well as a model theoretic application of my previous paper Sd- Topology over the theory of a Model. The main intention of this article is to proof and visualize the celebrated Gödel's completeness and incompleteness theorems in a different and lucid approach.
\end{abstract}

Keywords: Theory, Theorem, Proof, Kuratowski’s Closer Operator, Topology, Sd-Topology, Gödel's completeness and incompleteness theorems.

\section{INTRODUCTION}

This is a continuation and application of Sd-Topology over the theory of a Model (DOI: 10.17148/IARJSET.2020.71201) to provide a lucid and short proof of the famous Gödel's completeness and incompleteness theorems. Here as we are using Sd Topology so the conditions will be restricted as the cardinality of the universe of the working model should be card $\boldsymbol{U} \geq \boldsymbol{N}_{0}$ and also the cardinality of the working theory should be card(Th $\left.\boldsymbol{U}\right) \geq \operatorname{card}(\boldsymbol{U}$ ). The inspiration of this article also have a very deep connection with philosophy. The similar statements of the Gödel's incompleteness theorems have also been mentioned in the ancient Rigveda's 10/129th mandala as following,

नासदासीत्रो सदासीत्तदानीं नासीद्रजो नो व्योमा परो यत्।

किमावरीवः कुह कस्य शर्मन्नम्भः किमासीद्रहनं गभीरम् ॥ १॥

को अद्धा वेद क इह प्र वोचत्कुत आजाता कुत इयं विसृष्टिः |

अर्वाग्देवा अस्य विसर्जनेनाथा को वेद यत आबभूव ॥६॥

इयं विसृष्टिर्यत आबभूव यदि वा दधे यदि वा न।

यो अस्याध्यक्षः परमे व्योमन्त्सो अड्ग वेद यदि वा न वेद ॥७॥

The meaning of these Slokas are:

1. Then even non-existence was not there, nor existence, There was no air then, nor the space beyond it. What covered it? Where was it? In whose keeping? Was there then cosmic fluid, in depths unfathomed.

6. But, after all, who knows, and who can say whence it all came, and how creation happened? the gods themselves are later than creation, so who knows truly whence it has arisen?

7. Whence all creation had its origin, the creator, whether he fashioned it or whether he did not, the creator, who surveys it all from highest heaven, he knows - or maybe even he does not know. 


\section{DOI: $10.17148 /$ IARJSET.2021.8109}

DEFINITION : If $\mathcal{U}$ is a model for the language $\mathscr{L}$, the theory of $\mathcal{U}$ is denoted by $\mathbf{T h} \mathcal{U}$, is defined to be the set of all sentences of $\mathscr{L}$ (i.e formulas with no free variables) which are true in $\mathcal{U}$. So Th $\mathcal{U}=\{\sigma$ of $\mathscr{L}: \mathcal{U} \vDash \sigma\}$

For example if $\mathscr{L}=\{\langle,+,, 0,1\}$ then $\mathbf{T h} \mathbb{R}$ is famous as the name Real Analysis. Similarly over the same language $\mathbf{T h} \mathbb{Z}$ is known as number theory again for $\mathscr{L}=\{+, \cdot, 0,1\} \mathbf{T h} \mathbb{C}$ is renowned as complex analysis.

[ Note: here we denote the models for a given language as italic notations as the universe of the model i.e $\mathbb{R}=\langle\mathbb{R}, I\rangle$ where $I$ is the respective interpretation function, also further we indicate the cardinality of a model as the cardinality of its universe i.e card $\mathbb{R}$ means card $\mathbb{R}=c]$

\section{Construction of $S d$-topology on $\operatorname{Th} \mathcal{U}$ (When card $\left.\mathcal{U} \geq \boldsymbol{\aleph}_{0}\right)$ :}

Lets define a mapping $k: \mathrm{P}(\mathbf{T h} \boldsymbol{U}) \rightarrow \mathrm{P}(\mathbf{T h} \boldsymbol{U})$ as

$k(\mathrm{~A})=\mathrm{A}$ if card $\mathrm{A}<$ card $\boldsymbol{U} \quad$ [ where $\mathrm{A}$ is subset (i.e sub theory) of $\mathbf{T h} \boldsymbol{U}$ ]

$k(\mathrm{~A})=\mathbf{T h} \boldsymbol{U}$ if card $\mathrm{A} \geq$ card $\mathcal{U}$

It's easy to verify that $\mathrm{k}$ is a Kuratowski's Closer Operator

since $k(\varnothing)=\varnothing, \mathrm{A} \subseteq k(\mathrm{~A}) \& k(k(\mathrm{~A}))=k(\mathrm{~A}) ; \forall \mathrm{A} \subseteq \mathbf{T h} \mathcal{U}$ and $k(\mathrm{~A} \mathrm{U} \mathrm{B})=k(\mathrm{~A}) \mathrm{U} k(\mathrm{~B}) ; \forall \mathrm{A}, \mathrm{B} \subseteq \mathbf{T h} \mathcal{U}$

LEMMA: Let $\mathrm{k}$ be a Kuratowski's Closer operator on a set $\mathrm{X}$. Then there is a unique topology $\tau$ on $\mathrm{X}$ such that $\mathrm{k}(\mathrm{A})=\overline{\mathrm{A}}$ ; in $(\mathrm{X}, \tau) \forall \mathrm{A} \subseteq \mathrm{X}$ where $\overline{\mathrm{A}}$ is the closer of $\mathrm{A}$.

Proof: Let $\sum=\{\mathrm{k}(\mathrm{A}): \mathrm{A} \subseteq \mathrm{X}\}$ since $\mathrm{k}(\mathrm{k}(\mathrm{A}))=\mathrm{k}(\mathrm{A}), \forall \mathrm{A} \subseteq \mathrm{X}$

so we can treat $\sum=\{\mathrm{A} \subseteq \mathrm{X}: \mathrm{k}(\mathrm{A})=\mathrm{A}\}$, Obviously $\emptyset \in \sum$ and $\mathrm{X} \in \sum$

Again if $A, B \in \sum$ then as $\left.k(A) B\right)=k(A) U k(B)=A U B$, so $A U B \in \sum$ thus $\sum$ is closed under finite union. Again if $A_{i} \in \sum$ then as $\bigcap_{i} A_{i} \subseteq A_{i}$ so by the property of $k$,

$\mathrm{k}\left(\bigcap_{\mathrm{i}} \mathrm{A}_{\mathrm{i}}\right) \subseteq \mathrm{k}\left(\mathrm{A}_{\mathrm{i}}\right)=\mathrm{A}_{\mathrm{i}}$ therefore $\bigcap_{\mathrm{i}} \mathrm{A}_{\mathrm{i}} \subseteq \mathrm{k}\left(\cap_{\mathrm{i}} \mathrm{A}_{\mathrm{i}}\right) \subseteq \bigcap_{\mathrm{i}} \mathrm{A}_{\mathrm{i}}$ and $\mathrm{k}\left(\cap_{\mathrm{i}} \mathrm{A}_{\mathrm{i}}\right)=\bigcap_{\mathrm{i}} \mathrm{A}_{\mathrm{i}}$

thus $\cap_{i} A_{i} \in \sum$ so it's also closed under arbitrary intersection. Therefore $\tau$ is defined as

$\tau=\left\{\mathrm{V}: \mathrm{X}-\mathrm{V} \in \sum\right\}$, surly a topology on $\mathrm{X}$ with $\sum$ is the set of all closed sets in $(\mathrm{X}, \tau)$.

Now $\bar{A} \subseteq k(A)$ since $k(A)$ is a closed set containing $A$. Again $\bar{A} \subseteq k(\bar{A}) \subseteq k(k(A))=k(A)$.

But $\mathrm{k}(\mathrm{A}) \subseteq \mathrm{k}(\overline{\mathrm{A}})$ so we get $\mathrm{k}(\mathrm{A})=\overline{\mathrm{A}}$.

For uniqueness if $\tau_{1}$ and $\tau_{2}$ are two topologies on $\mathrm{X}$ such that $\mathrm{Cl} \tau_{1}(\mathrm{~A})=\mathrm{Cl} \tau_{2}(\mathrm{~A}), \forall \mathrm{A} \subseteq \mathrm{X}$.

Then $\left\{\mathrm{Cl} \tau_{1}(\mathrm{~A}): \mathrm{A} \subseteq \mathrm{X}\right\}=\left\{\mathrm{Cl} \tau_{2}(\mathrm{~A}): \mathrm{A} \subseteq \mathrm{X}\right\}$ so $\tau_{1}=\tau_{2}\left(\right.$ Where $\mathrm{Cl} \tau_{1}(\mathrm{~A})=\overline{\mathrm{A}}$ in $\left.\left(\mathrm{X}, \tau_{1}\right)\right)$

( Q.E.D)

Now with the help of this lemma and the Kuratowski's Closer Operator ' $k$ ' what we had defined earlier, we can construct a unique topology over $\mathbf{T h} \mathcal{U}$. Where $k(\mathrm{~A})=\overline{\mathrm{A}}, \forall \mathrm{A} \subseteq \mathbf{T h} \mathcal{U}$. We called that topology is the $\boldsymbol{S d}$-topology on $\mathbf{T h} \mathcal{U}$. We are denoting that topology further as $\tau_{\mathrm{Sd}}$. Another thing to note that if $\operatorname{card} \boldsymbol{\mathcal { U }}<\boldsymbol{\aleph}_{\mathbf{0}}$ or $\operatorname{card}(\mathrm{Th} \boldsymbol{U})<\operatorname{card}(\boldsymbol{U})$ then $\left(T h \boldsymbol{U}, \tau_{S d}\right)$ will be very boring and elementary so in general we consider $\left(T h \boldsymbol{U}, \tau_{S d}\right)$ with card $\boldsymbol{U} \geq \boldsymbol{\aleph}_{\mathbf{0}}$ and card(Th $\left.\boldsymbol{U}\right) \geq$ $\operatorname{card}(\mathcal{U})$.

\section{Some Properties of the topological space $\left(\mathrm{Th} \mathcal{U}, \tau_{\mathrm{Sd}}\right):$}

1) In $\left(T h \mathcal{U}, \tau_{S d}\right)$ any Sub Theory ( i.e non trivial subsets of a theory) containing equal or more sentences than the cardinality of the universe of the respective model, then it's closer is itself the theory. In other words we can say that in $\left(T h \mathcal{U}, \tau_{S d}\right)$ if $A \subseteq \boldsymbol{T h} \mathcal{U}$ and $\operatorname{card}(A) \geq \operatorname{card}(\mathcal{U})$ then $A$ is dense in $\left(T h \mathcal{U}, \tau_{S d}\right)$.

Proof: Its trivial to check that as the operator defined as $k(\mathrm{~A})=\mathbf{T h} \mathcal{U}$ if card $\mathrm{A} \geq \operatorname{card} \mathcal{U}$. Also as in $\left(T h \mathcal{U}, \tau_{s d}\right), \mathrm{k}(\mathrm{A})=$ $\overline{\mathrm{A}}, \forall \mathrm{A} \subseteq \mathbf{T h} \mathcal{U}$. So whenever card $\mathrm{A} \geq$ card $\mathcal{U}$ then $\overline{\mathrm{A}}=\boldsymbol{T h} \boldsymbol{U}$. (Q.E.D)

2) In $\left(T h \mathcal{U}, \tau_{S d}\right)$ any Sub Theory A with card $(A)<\operatorname{card}(\mathcal{U})$ is closed. Also vise-versa. 


\section{International Advanced Research Journal in Science, Engineering and Technology}

Vol. 8, Issue 1, January 2021

\section{DOI: $10.17148 /$ IARJSET.2021.8109}

DEFINITION: A sentence $\sigma \in T h \mathcal{U}$ is said to be a Theorem iff there exist a convergent sequence of different sentences $\left\{\sigma_{\mathrm{n}}\right\}_{\mathrm{n} \in \mathrm{N}}\left(\mathrm{i} . \mathrm{e}\right.$ steps) in $T h \mathcal{U}$, which converges to $\sigma$ in $\left(T h \mathcal{U}, \tau_{S d}\right)$. Then the set $\left\{\sigma_{\mathrm{n}}: \mathrm{n} \in \mathbb{N}\right\} U$

$\{\sigma\}$ is called a proof.

Basically as we know that the last line of a proof is called a Theorem.

$\underline{\text { Theorem: }}$ A sentence is a Theorem iff it can be proved using finite number of steps.

To proof this theorem using finite steps we have to discuss some lemmas in $\left(T h \mathcal{U}, \tau_{S d}\right)$

LEMMA 1: In $\left(T h \mathcal{U}, \tau_{S d}\right)$ if card $\boldsymbol{U}=\boldsymbol{\aleph}_{0}$ a sequence is convergent iff its semi-constant. ( i.e exactly one term of the sequence repeated infinitely many times)

Proof: In (Th $\left.\boldsymbol{U}, \tau_{\mathrm{Sd}}\right)$ if card $\boldsymbol{U}=\boldsymbol{\aleph}_{\mathbf{0}}$ the space is a cofinite space and the above lemma is evident there also that sequence converges to that particular term(point) always.We can find this lemma in almost all general topology book.

LEMMA 2: In $\left(\mathrm{Th} \boldsymbol{U}, \tau_{\mathrm{Sd}}\right)$ if card $>\boldsymbol{\aleph}_{\mathbf{0}}$ a sequence is convergent iff its eventually constant.

Proof: Let $\left\{\sigma_{\mathrm{n}}\right\}_{\mathrm{n} \in \mathrm{N}}$ be a non (eventually) constant sequence in $\left(\mathrm{Th} \boldsymbol{u}, \tau_{\mathrm{Sd}}\right)$ converges to $\sigma \in \mathrm{Th} \boldsymbol{u}$.

So $\sigma \notin\left\{\sigma_{\mathrm{n}}: \forall \mathrm{n} \in \mathbb{N}\right\}$ but the set $\left\{\sigma_{\mathrm{n}}: \mathrm{n} \in \mathbb{N}\right\}$ is countable, so its closed in (Thu, $\tau_{\mathrm{Sd}}$ ).

Then Thu- $\left\{\sigma_{n}: n \in \mathbb{N}\right\}$ is a neighbourhood of $\sigma$ which contains no $\sigma_{n}$, which is a contradiction.

The converse of the lemma is obvious.

Proof of the theorem: If ( $\mathrm{Th} \boldsymbol{U}, \tau_{\mathrm{Sd}}$ ) is with card $\boldsymbol{U}=\boldsymbol{\aleph}_{\mathbf{0}}$ then from the lemma 1 we can see that there any sequence is convergent iff its semi-constant. But as the definition of Theorem its proof cannot repeat any of its sentences. Thus for this case the proof should be finite. The converse is also true as by the lemma1. Now if $\left(\mathrm{Th} \boldsymbol{U}, \tau_{\mathrm{Sd}}\right)$ with card $>\boldsymbol{\aleph}_{\mathbf{0}}$ then by lemma2 the proof of any theorem should be an eventually constant sequence of sentences. With the same logic as before the proof should be finite. Again by lemma 2 its converse is also true. (Q.E.D)

\section{Gödel's Completeness Theorem:}

A theory is consistent iff it is satisfiable.

The logical statement of this theorem is $\mathscr{L} \vDash \sigma$ iff $\mathscr{L} \vdash \sigma$.

Stating the theorem in lucid words, if $\sigma \epsilon$ Th $\boldsymbol{U}$ for every model $\boldsymbol{U}$ over a language $\mathscr{L}$ then $\sigma$ is deducible (i.e provable using finite no. steps). Logically speaking a sentence of a theory is a syntactic consequence iff its a semantic consequence.

For example the theorem says that, when a sentence is shown to be provable from the axioms of ring theory by considering an arbitrary ring and showing that the sentence is satisfied by that ring.

Proof: We want to prove the following theorem involving the Sd topology as defined by us and since there is some restrictions as in general we considered $\left(T h \mathcal{U}, \tau_{S d}\right)$ with card $\boldsymbol{U} \geq \boldsymbol{N}_{\mathbf{0}}$ and card(Th $\left.\boldsymbol{U}\right) \geq$ card $(\boldsymbol{U})$. Thus its evident to say that we will restrict the proof of the theorem over the mentioned conditions. It's evident to see that its a corollary of the previous theorem. Since any sequence in $\left(T h \mathcal{U}, \tau_{S d}\right)$ with card $\boldsymbol{U} \geq \boldsymbol{N}_{\mathbf{0}}$ and $\operatorname{card}(\mathrm{Th} \boldsymbol{U}) \geq$ card $(\boldsymbol{U})$ is convergent iff its eventually constant. Thus if $\sigma \in \mathrm{Th} \boldsymbol{u}$ then its deducible.

Conversely, Let $\sigma$ is deducible in Th $\mathcal{u}$. Then there is an eventually constant sequence $\left\{\sigma_{\mathrm{n}}\right\}$ in $\left(T h, \tau_{S d}\right)$ which terminates in $\sigma$. So $\left\{\sigma_{1}, \sigma_{2}, \sigma_{3 \ldots} \sigma\right\}$ is a closed subset of $\left(T h \mathcal{U}, \tau_{S d}\right)$.

Thus surely $\sigma \in$ Thu. [ Q.E.D] The converse of the theorem is called Soundness.

Axioms: For axioms we indicate about the Non logical axioms or Postulates. Thus, a set of axioms is an elementary basis for a formal logic system that together with the rules of inference define a deductive system. But in our following field of discussion we will define Axioms through the topological spectacles. 


\section{DOI: $10.17148 /$ IARJSET.2021.8109}

Definition: Let $\left(T h \mathcal{U}, \tau_{S d}\right)$ with card $\boldsymbol{U} \geq \boldsymbol{\aleph}_{\mathbf{0}}$ and $\operatorname{card}(\mathrm{Th} \boldsymbol{U}) \geq \operatorname{card}(\boldsymbol{U})$ and $\mathcal{B}$ be a minimal open base of Th $\mathcal{U}$. Let $\mathcal{B}$

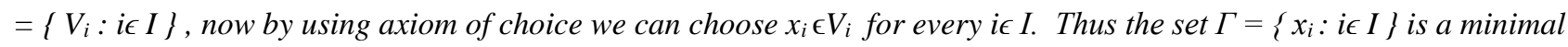
dense subset of $T h \mathcal{U}$. Such subsets are called set of Axioms for Th $\mathcal{U}$.

Theorem: Set of Axioms is a minimal dense subset of (Th $\left.\boldsymbol{U}, \tau_{S d}\right)$ with card $\boldsymbol{U} \geq \boldsymbol{\aleph}_{\mathbf{0}}$ and card $($ Th $\boldsymbol{U}) \geq \operatorname{card}(\boldsymbol{U})$.

Proof: As we know that for $\operatorname{card}(\boldsymbol{U})=\boldsymbol{\aleph}_{\mathbf{0}} ;\left(T h \boldsymbol{U}, \tau_{S d}\right)$ is equivalent to the cofinite topology whereas for card $(\operatorname{Th} \boldsymbol{U})=\boldsymbol{\aleph}_{1}$ ; (Th $\left.\mathcal{U}, \tau_{S d}\right)$ is equivalent to the cocountable topology and both of them are not $2^{\text {nd }}$ countable i.e they do not have any countable open base. So the set of axioms i.e $\Gamma=\left\{x_{i}: i \epsilon I\right\}$ are obviously uncountable there. Since they are derived from a minimal base using injective choice functions so surly such sets are minimal of their kind. Again as card $\Gamma \geq \boldsymbol{c a r d} \boldsymbol{U}=$ $\boldsymbol{\aleph}_{\mathbf{0}}$ or $\boldsymbol{\aleph}_{\mathbf{1}}$ so surly such $\Gamma$ are dense in $\left(T h \boldsymbol{U}, \tau_{S d}\right)$. Now we would like to extend our theorem for higher cardinals. Since our experiment runs over the set of infinite cardinals i.e $\left\{\boldsymbol{\aleph}_{\mathbf{0}}, \boldsymbol{N}_{\mathbf{1}}, \boldsymbol{\aleph}_{\mathbf{2}} \ldots\right\}$ which is a countably ordered set like $\mathbb{N}$ so we can use mathematical induction here (Because continuum hypothesis and ZFC are taken for granted ). Let $\left(T h \boldsymbol{U}, \tau_{S d}\right)$

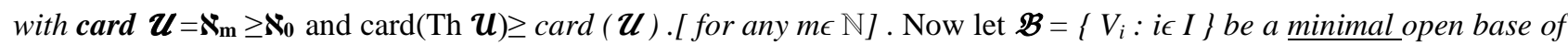
$T h \mathcal{U}$. Suppose card $\mathscr{B}<\boldsymbol{\aleph}_{\mathrm{m}}$ but card $\left(\mathrm{Th} \boldsymbol{U}-V_{i}\right)<$ card $T h \boldsymbol{U} \leq \boldsymbol{\aleph}_{\mathrm{m}}$. For every $V_{i} \in \mathfrak{B}$. Now by cardinal arithmetic $\cup_{i \epsilon I} \mathrm{Th}$ $\mathcal{U}-V_{i}<\boldsymbol{\aleph}_{\mathbf{m}}$ also $\bigcup_{i \epsilon I}\left(\mathrm{Th} \boldsymbol{U}-V_{i}\right)$ is non empty. So Th $\boldsymbol{U}-\cup_{i \epsilon I}\left(\right.$ Th $\left.\mathcal{U}-V_{i}\right)$ is open and contained in every $V_{i} \epsilon \mathfrak{B}$. Which is a contradiction. So card by mathematical induction card $\mathscr{B}=\boldsymbol{N}_{\mathbf{m}} \geq \boldsymbol{\aleph}_{\mathbf{0}}$ and card(Th $\left.\mathcal{U}\right) \geq \operatorname{card}(\mathcal{U})$. [ for any $m \epsilon$ $\mathbb{N}]$ Thus $\Gamma=\left\{x_{i}: i \epsilon I\right\}$ will always be dense in $\left(\right.$ Th $\left.\boldsymbol{U}, \tau_{S d}\right)$ since card $\Gamma=\operatorname{card} \mathfrak{B}=\operatorname{card}(\boldsymbol{U})$.

Property: From the previous theorem its obvious that the spaces(Th $\left.\boldsymbol{U}, \tau_{S d}\right)$ with card $\boldsymbol{U} \geq \boldsymbol{\aleph}_{\mathbf{0}}$ and card $($ Th $\boldsymbol{U}) \geq \operatorname{card}(\boldsymbol{U})$ are not $1^{\text {st }}$ countable too. So $\mathrm{A} \subseteq \mathbf{T h} \mathcal{U}$ with $\overline{\mathrm{A}}=\boldsymbol{T h} \mathcal{U}$ and $\sigma \epsilon \boldsymbol{T h} \mathcal{U}$-A then its impossible to find a sequence in $A$ converging to $\sigma$.

\section{Corollary: The Gödel's incompleteness theorem(1 $\left.{ }^{\text {st }}\right)$.}

These results, published by Kurt Gödel in 1931, are important both in mathematical logic and in the philosophy of mathematics. The theorems are widely, but not universally, interpreted as showing that Hilbert's program to find a complete and consistent set of axioms for all mathematics is impossible.

Observe that from our previous theorem $\Gamma$ is dense in $\mathbf{T h} \boldsymbol{U}$. Now by previous property we can see that if $\sigma \epsilon \boldsymbol{T h} \boldsymbol{U}$ - $\Gamma$ then its impossible to find any sequence inside $\Gamma$ converging to $\sigma$. So $\sigma$ can not be proved or disproved using any set of axioms in $\mathbf{T h} \mathcal{U}$.

Formal Statement $\left(1^{\text {st }}\right)$ : Any consistent formal system $F$ within which a certain amount of elementary arithmetic can be carried out is incomplete; i.e., there are statements of the language of $F$ which can neither be proved nor disproved in $F$." (Raatikainen 2015

In general we can say that there exist at least one $\sigma \in \mathbf{T h} \mathbb{N}$ such that there doesn't exist any $\Gamma \subset \mathbf{T h} \mathbb{N}$ such that $\Gamma \vdash \sigma$ or $\Gamma \vdash \vdash \sigma$.

For any such consistent formal system, there will always be statements about natural numbers that are true, but that are unprovable within the system. The second incompleteness theorem, an extension of the first, shows that the system cannot demonstrate its own consistency.

Example: Goodstein's theorem is a statement about the natural numbers, proved by Reuben Goodstein in 1944, which states that every Goodstein sequence eventually terminates at 0 . Kirby and Paris showed that it is unprovable in Peano arithmetic.

\section{Difference between the two completeness:}

Note that, in spite of their names, one is not a negation of the other. 


\section{International Advanced Research Journal in Science, Engineering and Technology}

Vol. 8, Issue 1, January 2021

\section{DOI: $10.17148 /$ IARJSET.2021.8109}

A set of axioms is (syntactically, or negation-) complete if, for any statement in the axioms' language, that statement or its negation is provable from the axioms which is used in the incompleteness theorem. Where as deductive system is called complete if every logically valid formula is the conclusion of some formal deduction, and the completeness theorem for a particular deductive system is the theorem that it is complete in this sense which has been used in the completeness theorem.

\section{CONCLUSION}

The remarkable Gödel's completeness and incompleteness theorems are the milestones in proof theory as well as in the whole field of mathematics, logic and philosophy. But their proves are too much long and difficult to digest by non mathematicians especially the Henkin's proof of the completeness theorem. Thus here we tried to give an easy and short proof of these theorems by introducing a new topological aspect, however the proves are restricted and conditionally dependent.

\section{REFERENCES}

1. Gödel's Completeness and incompleteness theorems by Ben Chiken - University of Chicago

2. Sd -Topology over the theory of a Model (DOI: 10.17148/IARJSET.2020.71201)- S.Dasgupta

3. What is Mathematical Logic ? by J.N.Crossley et.al , Dover Publication

4. Fundamentals of Model Theory by William Weiss and Cherie D'Mello ( University of Toronto)

5. Raatikainen, Panu, "Gödel's Incompleteness Theorems", The Stanford Encyclopedia of Philosophy (Winter 2020 Edition)EdwardN. Zalta (ed.), URL $=\langle$ https://plato.stanford.edu/archives/win2020/entries/goedel-incompleteness/>. 